\title{
Laser-impact-induced splashing: an analysis of the splash crown evolution after Nd:YAG ns-pulse laser impact on a liquid tin pool
}

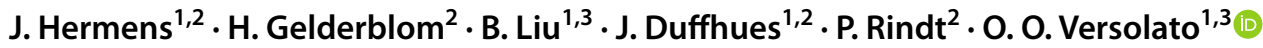

Received: 2 November 2020 / Accepted: 15 February 2021 / Published online: 28 February 2021

(c) The Author(s) 2021

\begin{abstract}
The splash created by intense laser pulse impact onto a liquid tin layer is studied experimentally using time-delayed stroboscopic shadowgraphy. An 8-ns infrared $(1064 \mathrm{~nm})$ laser pulse is focused onto a deep liquid tin pool. Various laser spot sizes $(70,120$, and $130 \mu \mathrm{m}$ in diameter) and various laser pulse energies (ranging $2.5-30 \mathrm{~mJ}$ ) are used, resulting in laser fluences of $\sim 10-1000 \mathrm{~J} / \mathrm{cm}^{2}$ inducing pronounced splashing. Specifically, we study the time evolution of the splash crown-width. The crown width expansion velocity is found to be linearly dependent on the laser energy, and independent of the focal spot size. A collapse of all crown width evolution data onto a single master curve confirms that the hydrodynamic evolution of our laser-impact-induced splash is equivalent to droplet-impact-induced splashing. Laser-impact splashing is particularly relevant, e.g. for high-brightness laser-assisted discharge-produced plasma and laser-produced plasma sources of extreme ultraviolet light for nanolithography.
\end{abstract}

\section{Introduction}

Splashing frequently occurs in nature [1,2], for example, as resulting from impact of raindrops on a pool. Such droplet impact onto a liquid pool may result in the formation of a liquid crown and in the subsequent breakup of the crown rim into small droplets - the 'splash' (as shown in Fig. 1a). The dynamics of this splash depends on the impact velocity, liquid drop size, and the fluid properties [2]. Splashing can also be generated without a droplet impactor, by, e.g. the impact of an air stream [3] or, as we show in this paper, from the plasma pressure-impulse induced by intense laser pulses impinging on liquid pools (as illustrated in Fig. 1b). Comparing Fig. 1a and b, we observe clear similarities between the two splashes. The splashes evolve very similarly despite of the length and time scales being very different $(\sim 0.1 \mathrm{~mm}$

O. O. Versolato

o.versolato@arcnl.nl

1 Advanced Research Center for Nanolithography (ARCNL), 1098 XG Amsterdam, The Netherlands

2 Department of Applied Physics, Eindhoven University of Technology, Den Dolech 2, 5600 MB Eindhoven, The Netherlands

3 Department of Physics and Astronomy, and LaserLaB, Vrije Universiteit Amsterdam, De Boelelaan 1081, 1081 HV Amsterdam, The Netherlands vs $\mathrm{mm}$ and $\sim \mu$ s vs $\mathrm{ms}$ ) highlighting the hydrodynamic similarity of the dynamics after the liquid is set in motion, be it by droplet impact or by a laser pulse.

Laser-impact-induced splashing of metals is of interest for a variety of industrial processes, considering the risk of process contamination from debris formed by splashing. For example, in laser-based micromachining applications, Willis et al. studied the melt zone formation and subsequent droplet generation after $\sim$ ns laser impact on thin chromium films [5]. Yilbas et al. investigated the laser heating and phase change process of steel and the related recoil pressures [6]. Ben-Yakar et al. analysed $\sim$ fs laser impact on glass surfaces, simulating the fluid flow of the melt zone and studying the morphology of the resulting crater [7]. Popescu et al. used high-speed imaging to study the liquid-metal dynamics during laser welding and analysed the spatter formation [8].

In the discipline of nanolithography, extreme ultraviolet (EUV) light is used to produce the next generation of computer chips. A laser produced plasma (LPP) generated from liquid tin microdroplets emits the required EUV light near $13.5 \mathrm{~nm}$ wavelength [9]. Variations on this concept are however also considered. Koshelev et al. devised an apparatus using a rotating drum covered by a liquid tin film, onto which a laser is shot to create EUV light. The rotation is foreseen to prevent the splash-generated fragments from reaching and contaminating optic elements [10]. Pawlowski et al. hold a patent on the concept of using rotating wheels, 


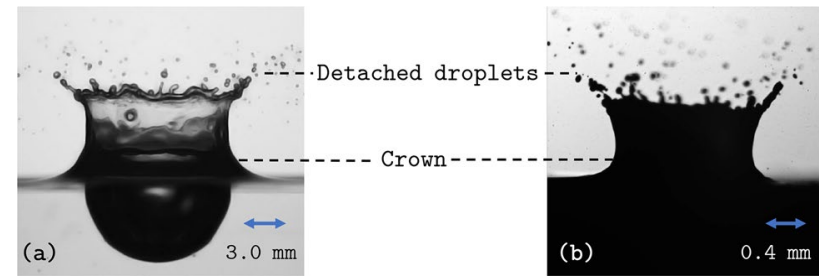

Fig. 1 Splashing, a crown and detached droplets are visible, resulting from a droplet impact (figure courtesy of A. Bisighini [4]) and $\mathbf{b}$ from laser pulse impact ( $30 \mathrm{~mJ}, 70 \mu \mathrm{m}$ case, see below)

covered with liquid tin, to produce EUV light from a laserassisted discharge plasma (LDP). In LDP sources, $\sim$ ns laser pulse impact (also $\sim$ ps pulses have been studied by Beyene et al. [11] for this purpose) ignites a first plasma, as well as a corresponding splash, between the rotating wheels serving as electrodes. These wheel electrodes subsequently discharge and produce a hot and dense tin plasma [12]. Despite the relevance of splashing for this wide field of application, no fundamental studies related to laser-impact-induced splashing on a liquid pool have yet entered the literature.

Here, we present an experimental study of laser-impactinduced splashing on a deep pool of liquid tin. Specifically, we study the time evolution of the splash crown width in detail using time-delayed stroboscopic shadowgraphy, varying the parameters of the laser impact under conditions relevant, e.g. for laser-assisted discharge plasma devices.

\section{Experiment}

In Fig. 2, an overview of the experimental setup is presented. A $1064 \mathrm{~nm}$ Nd:YAG laser pulse, with a full width half maximum (FWHM) pulse length of $8 \mathrm{~ns}$, is focused by a positive lens with a focal length $f=600 \mathrm{~mm}$. The laser energy can be adjusted with a half-wave plate and a thin-film polarizer. The focal spot size can be changed by adjusting the aperture behind a beam expander. A beam tower is used to enable the laser pulse to enter via the top window of the low pressure vacuum chamber (kept at a $\sim 0.1$ mbar hydrogen pressure), as is shown in a schematic side view in Fig. $2 b$. The pressure was kept low enough to prevent the ambient gas from influencing the laser-plasma generation process (e.g. by unwanted air breakdown). The laser pulse is focused onto a 3-mm-thick, temperature-controlled liquid tin pool and impacts along its surface normal. The liquid tin has a density $\rho=7.0 \mathrm{~g} / \mathrm{cm}^{3}$ and a surface tension $\sigma=0.54 \mathrm{~N} / \mathrm{m}$. The tin is kept at a constant temperature, either at $300^{\circ} \mathrm{C}$ or $430{ }^{\circ} \mathrm{C}$, well above the melting point of $232{ }^{\circ} \mathrm{C}$.

The dynamics of the liquid tin splash crown resulting from laser pulse impact are captured by means of stroboscopic shadowgraphy imaging [13]. This method combines incoherent pulsed backlight-illumination at $560 \mathrm{~nm}$ wavelength with a CCD camera coupled to a longdistance microscope aligned parallel to the pool surface. A spatial resolution of approximately $5 \mu \mathrm{m}$ is obtained. A dye laser provides the pulsed backlighting with a pulse duration of 5 ns (FWHM). To capture an image of the splash, first, the Nd:YAG laser pulse impacts the liquid tin to start crown formation, after which the backlight pulse is generated, and the shadow of the splash is imaged onto the CCD via the microscope. The time delay between the Nd:YAG laser pulse impact and the backlight pulse is stepped through to image the splash over time as shown in Fig. 2c-h. Consequently, every captured frame comes from a separate and new splash.

Experiments are conducted for laser energies ranging 2.5-30 mJ, focused to circular, Gaussian spot sizes with diameters 70, 120, and $130 \mu \mathrm{m}$ (FWHM), with Rayleigh lengths ranging $\sim 0.4-1 \mathrm{~cm}$ depending on the focusing condition. The height and top width of the crown, as shown in Fig. 2d, are determined using a MATLAB image processing tool developed specifically for this research. The crown height is defined as the lowest point on the corrugated rim relative to the pool interface height. The top width is determined by finding the coordinates where a horizontal line on the crown height position crosses the outside boundary of the crown, depicted in Fig. 2d.

\section{Results}

In Fig. 2c-h, the morphology of a typical laser-impactinduced splash is shown for various times after laser pulse impact. First, the ns laser pulse impacts the liquid film and generates a plasma (see, e.g., Refs. [14, 15] for a detailed discussion). This plasma pressure $[13,15]$ pushes aside a layer of liquid which moves into and over the nearby unperturbed liquid. This motion causes a crown to be ejected from the pool surface on a $\sim \mu$ s timescale. The crown grows over time and droplets start to detach from the crown rim by a combination of Rayleigh-Taylor and Plateau-Rayleigh instabilities [16, 17] $(\sim 10 \mu \mathrm{s})$. A swell wave on the bottom of the crown develops $(\sim 100 \mu \mathrm{s})$. In addition, on a similar timescale the crown retracts back towards the film surface (not shown in Fig. 2c-h). This timescale of retraction is found to be dependent on laser pulse energy. Lastly, after retraction of the crown, a liquid jet appears from the center $(\sim 1 \mathrm{~ms})$, similar to Worthington jets formed during droplet-impact splashing [18]. The swell wave around $\sim 100 \mu \mathrm{s}$, along with a limited contrast, complicates the determination of the the bottom width of the crown. Therefore, the top width of the crown is used in the analysis that follows. The crown height can also be obtained in principle but its measurement is hampered by 


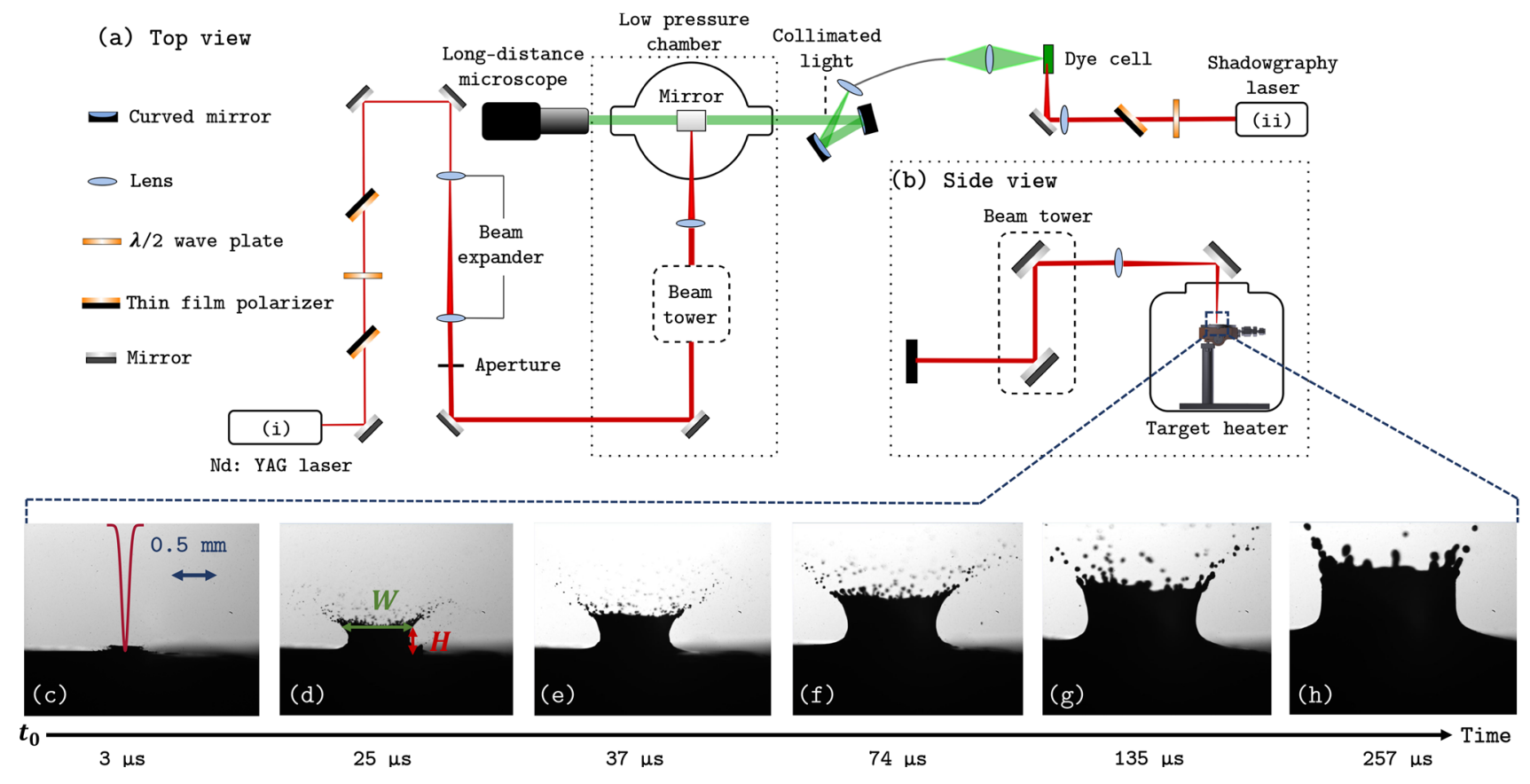

Fig. 2 a Schematic top view of the experimental setup. The experiment consists of two optical lines: (i) the Nd:YAG laser laser line is responsible for the laser ablation. The laser light is guided through a beam expander and aperture to control the beam diameter. Next, the pulse is focused on the liquid tin target shown in side view (b). The laser-impact-induced splash is imaged stroboscopically using the second optical line (ii) consisting of a dye cell that creates a collimated

the formation of ligaments and by the fragmentation processes. Such effects do not significantly affect the determination of the crown width. Accordingly, a study of the crown height is left for future work and we focus our studies on the evolution of the crown width.

In Fig. 3, the crown width is tracked over time for laser impacts with various energies and spot sizes. Figure $3 \mathrm{a}$ shows how the crown width evolution varies with laser energy $(2.5-30 \mathrm{~mJ})$, for a constant spot size of $70 \mu \mathrm{m}$ (FWHM). Clearly, the crown width significantly exceeds the laser beam spot size at all times shown. The crown width at any given time increases with laser energy. At early times ( $\lesssim 100 \mu \mathrm{s}$ ), the radial expansion speed of the crown clearly increases with laser pulse energy. After approximately $200 \mu \mathrm{s}$, the radial expansion speeds appear to converge to a common, constant value for all measured laser energies.

Figure $3 \mathrm{~b}$ shows the crown width as a function of time for various laser pulse energies $(5,10$, and $20 \mathrm{~mJ}$ cases) each for which the spot size was changed $(70,120$, and/or $130 \mu \mathrm{m}$ FWHM). The crown width and its dynamics appear to be independent of the spot size. Only at early time $(t \leq 10 \mu \mathrm{s})$ the spot size influences the crown size both in terms of initial velocity and apparent initial diameter (see inset Fig. 3c). $\sim$ 5ns-long 560-nm backlight pulse using a controlled time delay after laser pulse impact. The splash is imaged via a long-distance microscope. In (c-h) images for different time steps after laser impact of a $30 \mathrm{~mJ}$ laser pulse are displayed. The size of a $70 \mu \mathrm{m}$ Gaussian laser spot is indicated to scale in (c). The definitions of splash crown height $H$ and width $W$ are illustrated in (d)

These differences, however, quickly fade after $\sim 10 \mu \mathrm{s}$. Preliminary qualitative analysis of the images suggests that the size and number of droplets detached during the splash may vary with spot size. A more detailed study of any remaining differences between the laser-impact-induced splashes with differing spot sizes is left for future work.

To further interpret and explain the dependence of the evolution of the crown width on laser pulse energy, as well as the lack of any clear such dependence on laser spot size, we next introduce in our studies a dimensionless crown width $W^{*}=W / D$ and time $t^{*}=t U / D$ in analogy to droplet-impact studies, see e.g., Yarin et al. [2, 19]. Here, $D$ is the impactor length scale and $U$ the impactor velocity. It is shown in, e.g., Refs. [19-21] that the dimensionless width $W^{*}$ is independent of impactor size and velocity, i.e., $W^{*}=f\left(t^{*}\right)$. For the case of laser-impact-induced splashing, these length and velocity scales need to be inferred from the laser pulse properties.

First, the laser-impact length scale $D_{L}$ needs to be defined. The laser spot size would appear to be the natural choice for this length scale. However, the crown width dynamics appear to be independent on spot size, as shown in Fig. 3b, even though the laser fluence changes by more than a factor 

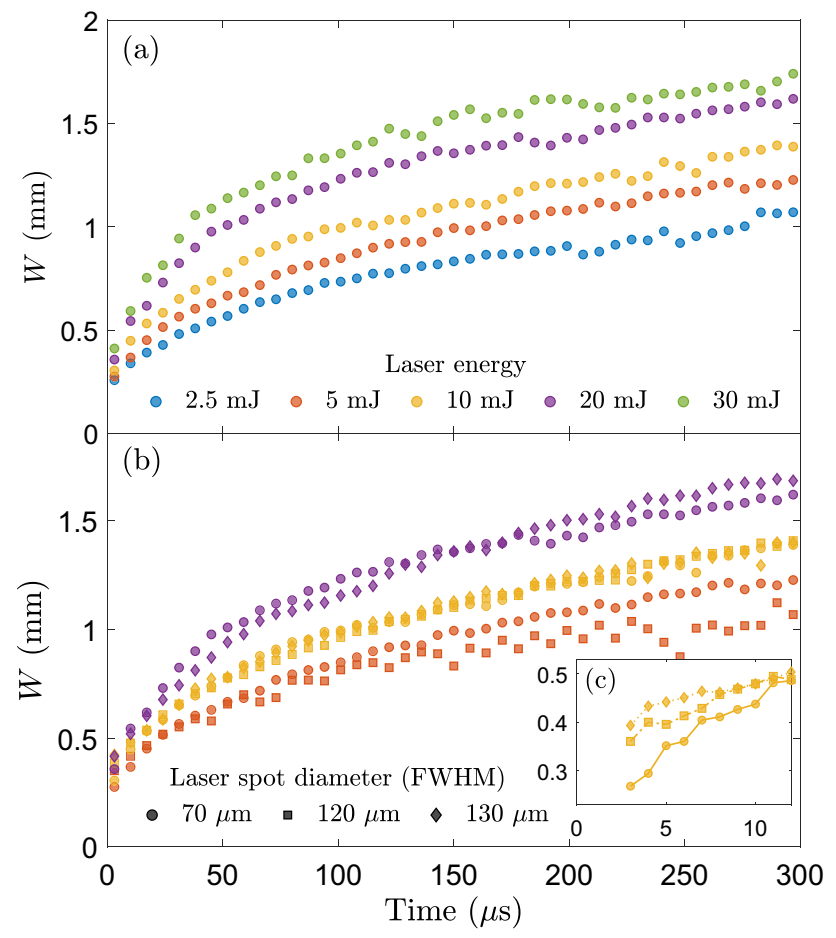

Fig. 3 Crown width evolution of a laser-impact-induced splash in time. a Results for a constant spot size of $70 \mu \mathrm{m}$ (FWHM) for various laser energies ranging 2.5-30 mJ. b Results for sets of energy $(5,10$, and $20 \mathrm{~mJ}$ cases) each for multiple spot diameters $(70,120$, and $130 \mu$ $\mathrm{m})$. c Early-time zoom-in of the $10 \mathrm{~mJ}$ case for the three spot sizes

three, comparing 70 to $130 \mu \mathrm{m}$ spot sizes. Alternatively, we study the axis intercepts of all measurements shown in Fig. 3 to obtain an initial crown width, extrapolating to the onset moment of laser pulse impact. We find that linear fits of the evolution of all crown widths over the first $\sim 20-40 \mu \mathrm{s}$ have $y$-axis intercepts ranging approximately $0.30-0.45 \mathrm{~mm}$. Considering this modest range and given the fact that no systematic dependence on laser spot size from the linear fits is found, we use $D_{L}=0.4 \mathrm{~mm}$ as a typical and constant length scale in the following. This length scale is significantly larger than the beam spot sizes used (i.e. 70 - $130 \mu$ $\mathrm{m})$. This apparent decoupling of laser spot size from the relevant typical length scales may be related to the initial dynamics directly following the laser impact and plasma expansion, which could not be sufficiently resolved in our experiments.

Second, a typical liquid velocity $U_{L}$ needs to be defined. To this end, we assume that the expanding plasma with pressure $P_{p}$ imparts a momentum over an area $A$. The plasma pressure scales with laser intensity as $\sim I^{\beta}$, where exponent $\beta$ typically varies between 0.5 and 1.0 depending on the experimental conditions $[13,15,22,23]$. For tin droplet targets hit by a Nd:YAG laser pulse, for instance, a power scaling of 0.6 was accurately established [13, 15]. For solid planar tin targets, hit by an energetic $\mathrm{CO}_{2}$ laser pulse, a larger power of $0.96 \pm 0.07$ was experimentally found by Lakatosh et al. [23]. As we are also dealing with a planar tin target and with similar laser fluences, we would expect a similar scaling of the total imparted momentum. Using $p \propto I^{0.96} A t_{p}$, with laser spot area $A$ and pulse length $t_{p}$, and combine this with $E=I A t_{p}$, we find

$p \propto I^{0.96} A t_{p}=\left(E / A t_{p}\right)^{0.96} A t_{p} \approx E$.

Here, the transferred momentum scales linearly with pulse energy to good approximation, $p \propto E$. Note that this scaling is independent of spot size, in line with our findings above related to the observed independence of the crown width dynamics on spot size. We next make the ansatz that the typical momentum scaling directly translates to a velocity scaling $U_{L} \propto E$ given the fact that the length scale $D_{L}$ remains unchanged and with it, by assumption, the displaced mass.

The scaling of the typical velocities $U_{L}$ with laser pulse energy $E$ is obtained directly from the experiments using the following procedure. We expect all measurements for the crown width to collapse onto a single curve according to the relation $W^{*}=f\left(t^{*}\right)$ following, e.g., Yarin et al. [19]. Therefore, we next minimize the differences between all the curves by varying $t^{*}$ through changing $U_{L}$, thus 'stretching' the horizontal axis to collapse the curves onto each other. Measurement data for $t<200 \mu \mathrm{s}$ are used for the fitting procedure. No collapse is expected for later times $t>200 \mu \mathrm{s}$ where the crown width velocities for all measurements appear to converge to a common and approximately constant velocity (as shown in Fig. 3) and other dynamics, such as those mediated by surface tension, are at play.

The resulting collapse of all data is shown in Fig. 4a and the associated relation of $U_{L}$ with laser pulse energy $E$, also obtained from the procedure, is shown in Fig. 4b. The excellent collapse of all data shown in Fig. 4a onto a single 'master' curve indicates that the scaling of the hydrodynamic response is well captured by a single parameter $U_{L}$. Furthermore, we note that the scaling of $U_{L}$ with pulse energy $E$ accurately follows a linear relation, in line with our predictions from Eq. (1). The a priori unknown pre-factors $C_{1}$ and $C_{2}$ in the linear relation $U_{L}=C_{1} E+C_{2}$ following Eq. (1) can be assigned by determining the absolute value of velocity $U_{L}$ directly from the experiment. This value is obtained from a linear fit to the early-time evolution $(20<t<40 \mu \mathrm{s})$ of the crown width of the $30 \mathrm{~mJ}$ example case, which gives a slope of $17 \mathrm{~m} / \mathrm{s}$. Therefore, we set $U_{L}=17 \mathrm{~m} / \mathrm{s}$ for the $30 \mathrm{~mJ}$ laser impact case. Applying this velocity as a calibration factor for the unknown pre-factors $C_{1}$ and $C_{2}$, we obtain $U_{L}[\mathrm{~m} / \mathrm{s}] \approx 0.5 E[\mathrm{~mJ}]+0.5$. This relation serves as the required input establishing the horizontal $t^{*}$ axis in Fig. 4a and sets the vertical $U_{L}$ axis in Fig. $4 \mathrm{~b}$. The relatively small $0.5 \mathrm{~m} / \mathrm{s}$ offset at $E=0 \mathrm{~mJ}$ may be explained considering that the relation between $U_{L}$ and $E$ cannot be expected to be 
perfectly linear especially towards the lower energies (close to the ablation threshold of liquid tin). The threshold of the formation of plasma, and the threshold of any accompanying hydrodynamic response of the liquid, was shown to be laser fluence dependent $[13,15]$ and to have a typical value of $\sim 5 \mathrm{~J} / \mathrm{cm}^{2}$. This puts the current experiments well above threshold, into the fully ablative regime [15]. We separately note that any scaling law with power $\beta<1$ gives rise to a finite positive $y$-axis offset when approximated with a linear function as in the current case.

The velocity magnitude can be compared with estimations made using the Navier-Stokes equation $\mathrm{d} P / \mathrm{d} x=\rho \mathrm{d} v / \mathrm{d} t$, with radial length scale $d x$, characteristic velocity $v$, and $t$ the time over which the pressure is applied. From the work of Lakatosh et al. [23] we obtain a pressure $P \approx 3 \times 10^{9} \mathrm{~Pa}$. This is done by extracting the recoil momentum at the relevant laser fluence (this fluence corresponds to our $30 \mathrm{~mJ}$ laser pulse energy example case above) and deriving the corresponding exerted pressure averaging of the laser spot size and pulse length as reported [23]. We note that the same order of magnitude for the pressure is obtained using the values from Kurilovich et al. [13, 15]. Using a $3 \times 10^{9} \mathrm{~Pa}$ pressure as input for the Navier-Stokes equation we obtain $v \sim 20 \mathrm{~m} / \mathrm{s}$ (averaging over our $8 \mathrm{~ns}$ pulse length $t_{p}$, inputting $0.4 \mathrm{~mm}$ as $2 d x$ and taking the liquid density of tin $\rho=7.0 \mathrm{~g} /$

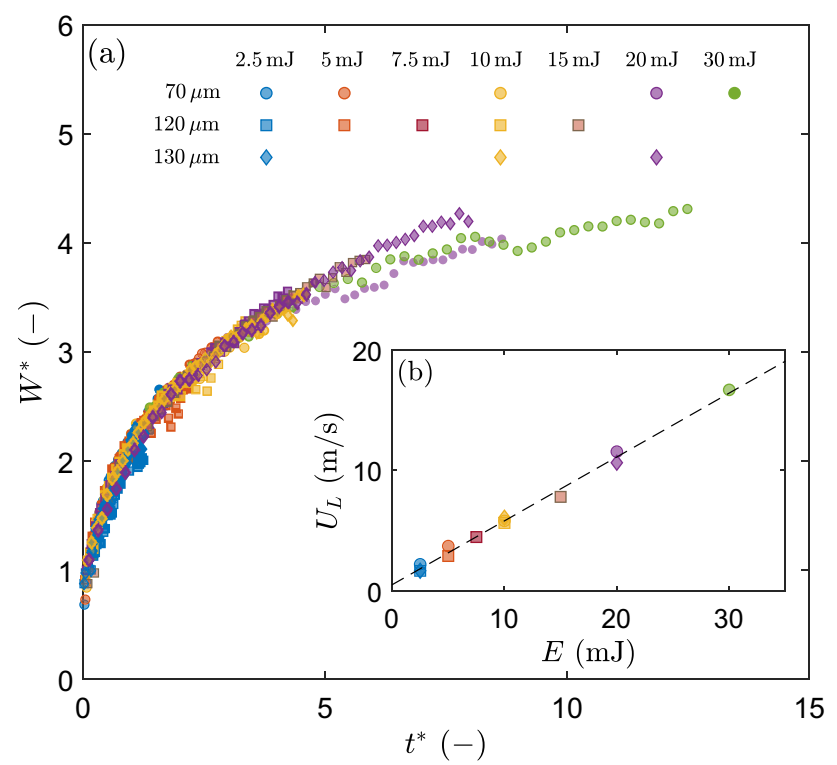

Fig. 4 a Collapse of the crown width over time for all laser-impactinduced splashing measurements, with laser pulse energies ranging $2.5-30 \mathrm{~mJ}$, and spot sizes of 70,120 , and $130 \mu \mathrm{m}$. This collapse is obtained by introducing a dimensionless parameter space where $W^{*}=W / D_{L}$ and time $t^{*}=t U_{L} / D_{L}$ (see main text). b Characteristic velocity $U_{L}$ extracted from the experimental data as a function of laser pulse energy $E$. The dashed line shows the result of a linear fit to the data $\mathrm{cm}^{3}$ ) in excellent agreement with the value obtained directly from the experiment.

For the impact of a droplet onto a liquid pool instead of a laser pulse, the subsequent crown dynamics is often described in terms of the ratio of the kinetic energy of the impactor (with known impact velocity $U$ and droplet diameter $D$ ) to its surface energy as captured by the Weber number We $=\rho D U^{2} / \sigma$ (see, e.g., Ref. [2]). Taking our observed typical length scale and inferred velocity for the $30 \mathrm{~mJ}$ example case as input for $D$ and $U$, respectively, we find $\mathrm{We} \approx 2000$. Even though this estimate is tentative, splash characteristics are indeed qualitatively similar comparing droplet-impact with laser-impact induced splashing in Fig. 1, where $\mathrm{We}=2207$ for the droplet-impact case [4].

\section{Conclusion}

The splash created from intense Nd:YAG laser pulse impact onto a deep pool of liquid tin was studied experimentally by time-delayed stroboscopic shadowgraphy. Various laser spot sizes $(70,120$, and $130 \mu \mathrm{m}$ in diameter) and laser pulse energies (ranging $2.5-30 \mathrm{~mJ}$ ) were used, resulting in laser fluences of $\sim 10-1000 \mathrm{~J} / \mathrm{cm}^{2}$. The time evolution of the crown width was studied in detail. Similar to droplet-impact induced splashing, we could collapse the data for the crown evolution (for all laser energies and spot sizes) onto a single master curve by the choice of appropriate length- and velocity scales. From this collapse, the expansion velocity of the crown width was found to be linearly dependent on laser energy, and to be independent of the laser focus spot size. This scaling behavior could be explained based on previously derived plasma pressure scaling relations.

Our analysis further suggests that the crown dynamics would also be independent of the pulse duration $t_{p}$ in the nanosecond pulse regime. Our studies of laser-impactinduced splashing is particularly relevant for high-brightness laser-assisted discharge-produced plasma (LDP) and laserproduced plasma (LPP) sources of extreme ultraviolet light for nanolithography, where similar laser pulse energies, spot sizes, and pulse lengths are used for the generation of plasma from liquid tin targets. We further note that 'impactor-free' liquid metal splashing such as in the current case is also a critical concern in fusion reactors of the tokamak type. Millisecond transients in the fusion plasma can deliver significant heat loads and pressure [24] to advanced liquid metal heat shields $[25,26]$ which may subsequently splash, potentially disrupting the plasma reactor. Preventive measures can be taken, such as the use of porous structure in which the liquid is constrained by capillary forces. Nevertheless, reliably preventing splashing in tokamaks is a critical issue for stable 
operation, and the design of mitigation schemes may benefit from further insights such as those presented in this work.

Acknowledgements The authors thank Niek Lopes Cardozo, Job Beckers, Anton Darhuber, and Laurens van Buuren for fruitful discussions. Part of this work has been carried out at the Advanced Research Center for Nanolithography (ARCNL), a public-private partnership of the University of Amsterdam (UvA), the Vrije Universiteit Amsterdam (VU), the Netherlands Organisation for Scientific Research (NWO) and the semiconductor equipment manufacturer ASML.

Open Access This article is licensed under a Creative Commons Attribution 4.0 International License, which permits use, sharing, adaptation, distribution and reproduction in any medium or format, as long as you give appropriate credit to the original author(s) and the source, provide a link to the Creative Commons licence, and indicate if changes were made. The images or other third party material in this article are included in the article's Creative Commons licence, unless indicated otherwise in a credit line to the material. If material is not included in the article's Creative Commons licence and your intended use is not permitted by statutory regulation or exceeds the permitted use, you will need to obtain permission directly from the copyright holder. To view a copy of this licence, visit http://creativecommons.org/licenses/by/4.0/.

\section{References}

1. A. Worthington, A study of Splashing. London: Longmans, Green. 129 pp (1908)

2. A.L. Yarin, Drop impact dynamics: splashing, spreading, receding, bouncing. Ann. Rev. Fluid Mech. 38, 159-192 (2006)

3. T. Theofanous, G. Li, On the physics of aerobreakup. Phys. Fluids 20(5), 052103 (2008)

4. A. Bisighini, Single and double drop impacts onto deep and thick liquid layers. $\mathrm{PhD}$ thesis, Università degli studi di Bergamo (2010)

5. D. Willis, X. Xu, Transport phenomena and droplet formation during pulsed laser interaction with thin films. J. Heat Transf. 122(4), 763-770 (2000)

6. B. Yilbas, S. Mansoor, Laser evaporative heating of surface: simulation of flow field in the laser produced cavity. J. Phys. D Appl. Phys. 39(17), 3863 (2006)

7. A. Ben-Yakar, A. Harkin, J. Ashmore, R.L. Byer, H.A. Stone, Thermal and fluid processes of a thin melt zone during femtosecond laser ablation of glass: the formation of rims by single laser pulses. J. Phys. D Appl. Phys. 40(5), 1447 (2007)

8. A.C. Popescu, C. Delval, M. Leparoux, Control of porosity and spatter in laser welding of thick $\mathrm{AlMg}_{5}$ parts using high-speed imaging and optical microscopy. Metals 7(11), 452 (2017)

9. O.O. Versolato, Physics of laser-driven tin plasma sources of euv radiation for nanolithography. Plasma Sources Sci. Technol. 28(8), 083001 (2019)

10. K. Koshelev, A. Vinokhodov, O. Yakushev, A. Yakushkin, D. Abramenko, A. Lash, M. Krivokorytov, Y. Sidelnikov, V. Ivanov, V. Krivtsun, et al., "Debris-free high-brightness light source based on lpp for actinic euv microscopy and metrology applications. In International Conference on Extreme Ultraviolet Lithography 2018, vol. 10809, p. 108091Q, International Society for Optics and Photonics (2018)

11. G.A. Beyene, I. Tobin, L. Juschkin, P. Hayden, G. O’Sullivan, E. Sokell, V.S. Zakharov, S.V. Zakharov, F. O'Reilly, Laserassisted vacuum arc extreme ultraviolet source: a comparison of picosecond and nanosecond laser triggering. J. Phys. D Appl. Phys. 49(22), 225201 (2016)
12. Z. Pawlowski, J. A. W. M. Corver, R. Mohede, Z. Zajaczkowski, and M. Kennis, Radiation source for generating short-wavelength radiation from plasma. US Patent 9,119,279 (2015)

13. D. Kurilovich, A.L. Klein, F. Torretti, A. Lassise, R. Hoekstra, W. Ubachs, H. Gelderblom, O.O. Versolato, Plasma propulsion of a metallic microdroplet and its deformation upon laser impact. Phys. Rev. Appl. 6(1), 014018 (2016)

14. M.M. Basko, V.G. Novikov, A.S. Grushin, On the structure of quasi-stationary laser ablation fronts in strongly radiating plasmas. Phys. Plasmas 22(5), 053111 (2015)

15. D. Kurilovich, M.M. Basko, D.A. Kim, F. Torretti, R. Schupp, J.C. Visschers, J. Scheers, R. Hoekstra, W. Ubachs, O.O. Versolato, Power-law scaling of plasma pressure on laser-ablated tin microdroplets. Phys. Plasmas 25(1), 012709 (2018)

16. E. Castillo-Orozco, A. Davanlou, P.K. Choudhury, R. Kumar, Droplet impact on deep liquid pools: Rayleigh jet to formation of secondary droplets. Phys. Rev. E 92(5), 053022 (2015)

17. V. Cullinan, D. Morton, J. Liow, N. Gray, et al., Splash formation from impinging liquid drops. In Process Industries Power the Pacific Rim: Sixth Conference of the Asia Pacific Confederation of Chemical Engineering; Twenty-first Australasian Chemical Engineering Conference; Official Proceedings of Combined Conference 1993, p. 43, Institution of Engineers, Australia (1993)

18. H. Zhao, A. Brunsvold, S.T. Munkejord, Investigation of droplets impinging on a deep pool: transition from coalescence to jetting. Exp. Fluids 50(3), 621-635 (2011)

19. A. Yarin, D. Weiss, Impact of drops on solid surfaces: self-similar capillary waves, and splashing as a new type of kinematic discontinuity. J. Fluid Mech. 283, 141-173 (1995)

20. I.R. Peters, D. van der Meer, J.M. Gordillo, Splash wave and crown breakup after disc impact on a liquid surface. J. Fluid Mech. 724, 553-580 (2013)

21. G. Cossali, M. Marengo, A. Coghe, S. Zhdanov, The role of time in single drop splash on thin film. Exp. Fluids 36(6), 888-900 (2004)

22. C. Phipps Jr., T. Turner, R. Harrison, G. York, W. Osborne, G. Anderson, X. Corlis, L. Haynes, H. Steele, K. Spicochi et al., Impulse coupling to targets in vacuum by $\mathrm{KrF}, \mathrm{HF}$, and $\mathrm{CO}_{2}$ single-pulse lasers. J. Appl. Phys. 64(3), 1083-1096 (1988)

23. B. Lakatosh, D. Abramenko, V. Ivanov, V. Medvedev, V. Krivtsun, K. Koshelev, A. Yakunin, Propulsion of a flat tin target with pulsed $\mathrm{CO}_{2}$ laser radiation: measurements using a ballistic pendulum. Laser Phys. Lett. 15(1), 016003 (2017)

24. J. Coenen, G. Arnoux, B. Bazylev, G. Matthews, A. Autricque, I. Balboa, M. Clever, R. Dejarnac, I. Coffey, Y. Corre, S. Devaux, L. Frassinetti, E. Gauthier, J. Horacek, S. Jachmich, M. Komm, M. Knaup, K. Krieger, S. Marsen, A. Meigs, P. Mertens, R. Pitts, T. Puetterich, M. Rack, M. Stamp, G. Sergienko, P. Tamain, V. Thompson, ELM-induced transient tungsten melting in the JET divertor. Nucl. Fusion 55, 023010 (2015)

25. M. Jaworski, S. Gerhardt, N. Morley, T. Abrams, R. Kaita, J. Kallman, H. Kugel, R. Majeski, D. Ruzic, Macroscopic motion of liquid metal plasma facing components in a diverted plasma. Nucl. Mater. 415(1), S985-S988 (2011)

26. P. Fiflis, M. Christenson, M. Szott, K. Kalathiparambil, D. Ruzic, Free surface stability of liquid metal plasma facing components. Nucl. Fusion 56, 106020 (2016)

Publisher's Note Springer Nature remains neutral with regard to jurisdictional claims in published maps and institutional affiliations. 